Paper to be presented at the ANS Topical Meeting on Robotics and Remote Systems, February 5-10, 1995, Monterey, California

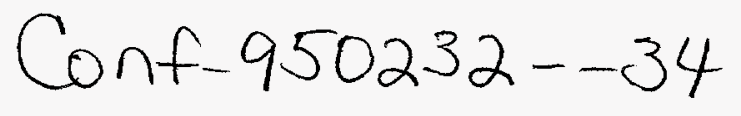

\title{
AUTOMATIC INSPECTION FOR REMOTELY MANUFACTURED FUEL ELEMENTS*
}

\author{
J. Reifman', K. S. Gibbs ${ }^{2}$, \\ J. E. Vitela', and R. W. Benedict ${ }^{2}$ \\ ${ }^{1}$ Reactor Analysis Division \\ ${ }^{2}$ Technology Development Division \\ Argonne National Laboratory \\ 9700 South Cass Avenue \\ Argonne, Illinois 60439
}

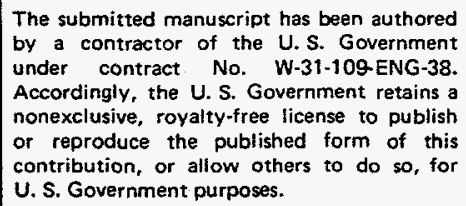

\section{DISCLAIMER}

This report was prepared as an account of work sponsored by an agency of the United States Government. Neither the United States Government nor any agency thereof, nor any of their employees, makes any warranty, express or implied, or assumes any legal liability or responsibility for the accuracy, completeness, or usefulness of any information, apparatus, product, or process disclosed, or represents that its use would not infringe privately owned rights. Reference herein to any specific commercial product, process, or service by trade name, trademark, manufacturer, or otherwise does not necessarily constitute or imply its endorsement, recommendation, or favoring by the United States Government or any agency thereof. The views and opinions of authors expressed herein do not necessarily state or reflect those of the United States Government or any agency thereof.

*Work supported by the U.S. Department of Energy, Nuclear Energy Programs under Contract W-31-109-ENG-38. 


\section{DISCLAIMER}

Portions of this document may be illegible in electronic image products. Images are produced from the best available original document. 


\section{AUTOMATIC INSPECTION FOR REMOTELY MANUFACTURED FUEL ELEMENTS}

J. Reifman

Argonne National Laboratory

Argonne, Illinois 60439

(708) 252-4685

jreifman@anl.gov
K. S. Gibbs

Argonne National Laboratory

Idaho Falls, Idaho 83403

(208) 533-7831

gibbs@anl.gov

\author{
J. E. Vitela \\ Argonne National Laboratory \\ Argonne, Illinois 60439 \\ (708) 252-8655 \\ vitela@salt.ra.anl.gov
}

R. W. Benedict

Argonne National Laboratory

Idaho Falls, Idaho 83403

(208) 533-7166

robert.benedict@anl.gov

\begin{abstract}
Two classification techniques, standard control charts and artificial neural networks, are studied as a means for automating the visual inspection of the welding of end plugs onto the top of remotely manufactured reprocessed nuclear fuel element jackets. Classificatory data are obtained through measurements performed on pre- and post-weld images captured with a remote camera and processed by an off-the-shelf vision system. The two classification methods are applied in the classification of 167 dummy stainless steel (HT9) fuel jackets yielding comparable results.
\end{abstract}

\section{INTRODUCTION}

Argonne National Laboratory (ANL) is developing an autonomous fuel element welding and visual inspection system as part of the fuel cycle demonstration for the Integral Fast Reactor project. This automated system performs the last few operations in the remote manufacturing of reprocessed nuclear fuel elements under a highly radioactive and inert gas environment. Stainless steel end plugs are welded onto the top of fuel element jackets which are then visually inspected on-line for possible defects and anomalies.

Automated on-line visual inspection is performed by coupling video images captured and processed by an offthe-shelf vision system to a decision-making advisory classifier. In this paper we present the preliminary results of the proposed inspection system in which two techniques, standard statistical process control and artificial neural networks, are used as classifiers. We describe the characteristics of each technique, their advantages and disadvantages, and the initial results obtained by applying them in the inspection of welded fuel elements.

\section{FUEL ELEMENT WELDING AND INSPECTION SYSTEMS}

The fuel element welding and visual inspection systems are located inside a "hot cell" of ANL's Fuel Cycle Facility (FCF) in Idaho. The hot cell is filled with argon gas with an anticipated average absorbed dose to the systems of approximately $100 \mathrm{~Gy} / \mathrm{hr}\left(10^{4} \mathrm{rad} / \mathrm{hr}\right)$. The components of the welding and inspection systems have been designed to withstand such high levels of radiation and to automatically perform four operations: (1) tag specific fuel elements with unique mixtures of xenon/krypton gas, (2) load end plugs into the top of fuel element jackets with outside diameter of $5.842 \mathrm{~mm}$, (3) weld end plugs to the top of fuel jackets, and (4) inspect welded fuel element jackets. ${ }^{1}$ In the following we briefly describe the components of the fuel welding and inspection systems and their operations.

\section{A. System Overview and Operation}

Figure 1 shows a schematic of the major in-cell components of the fuel element welding and inspection systems which are controlled by an out-of-cell computer system and touch-screen monitor at the Operator Control Station (OCS). The components of the system are stationed on top of a support table and are distributed around a removable Element Fabrication Magazine (EFM) at the center of the table which holds 36 fuel element jackets in the vertical position. The EFM fits into a rotate drive mechanism that rotates around the EFM's vertical axis allowing the fuel elements to be reached by the other components of the system which have horizontal and vertical linear slide capability.

Once the EFM loaded with fuel elements is in place, the sequence of gas tagging, end plug loading, welding, and inspection, commences with one fuel element at a time. First, a fuel element is positioned in front of the 


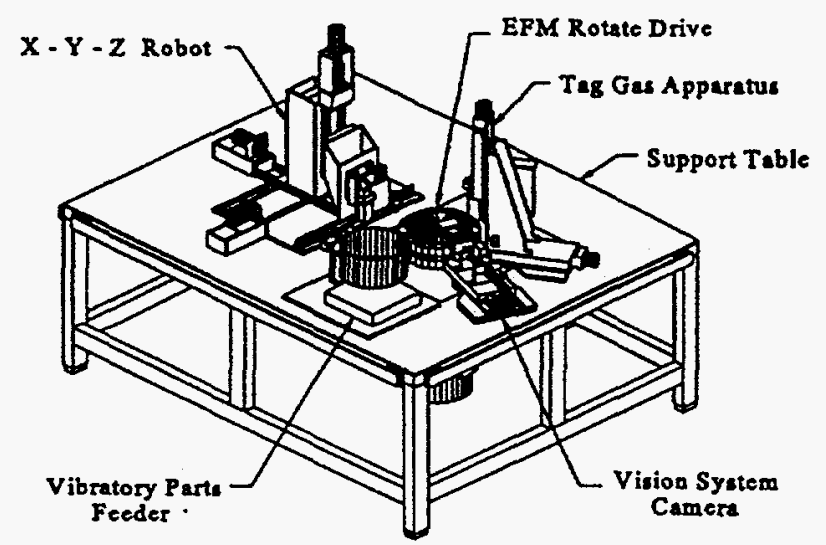

Figure 1. Major In-Cell Components of the Fuel Element Welding and Inspection System.

tag gas apparatus where a unique mixture of $x$ enon/krypton gas is injected into the open fuel element jacket. The tagging of the fuel jacket allows for future identification of the fuel element in case of fuel jacket breach. In parallel with the tag gas injection, the $X-Y-Z$ robot (capable of moving in the $X, Y$, and $Z$ directions) picks up an end plug from the vibratory parts feeder assembly and transfers it to the loading position. Second, the fuel element is rotated to the end plug loading position in front of the robot where the plug is inserted into the top of the element jacket.

Third, the fuel element is rotated and positioned in front of the vision system camera for pre-weld inspection, after which the element is rotated back where a gas tungsten arc welding device mounted on the $X-Y-Z$ robot welds the end plug onto the top of the element jacket. Fourth, the element is once again positioned in front of the vision system camera for post-weld inspection of possible defects and anomalies in the weld. This sequence of four operations is performed in approximately 6 minutes for each fuel element and continues until all 36 elements in the magazine have been welded.

\section{B. Vision System Components}

1. In-Cell Components. In addition to the radiation hardened tube-type camera illustrated in Fig. 1, the in-cell components of the vision system include three radiation hardened lights and a prism apparatus. Figure 2 shows the locations of these components in relation to a loaded EFM where the camera is on the right side of the figure behind one of the lights. The final configuration of the lighting scheme shown in the figure, with one back light and two directional lights, was arrived at after many trials. This configuration produces high-contrast binary

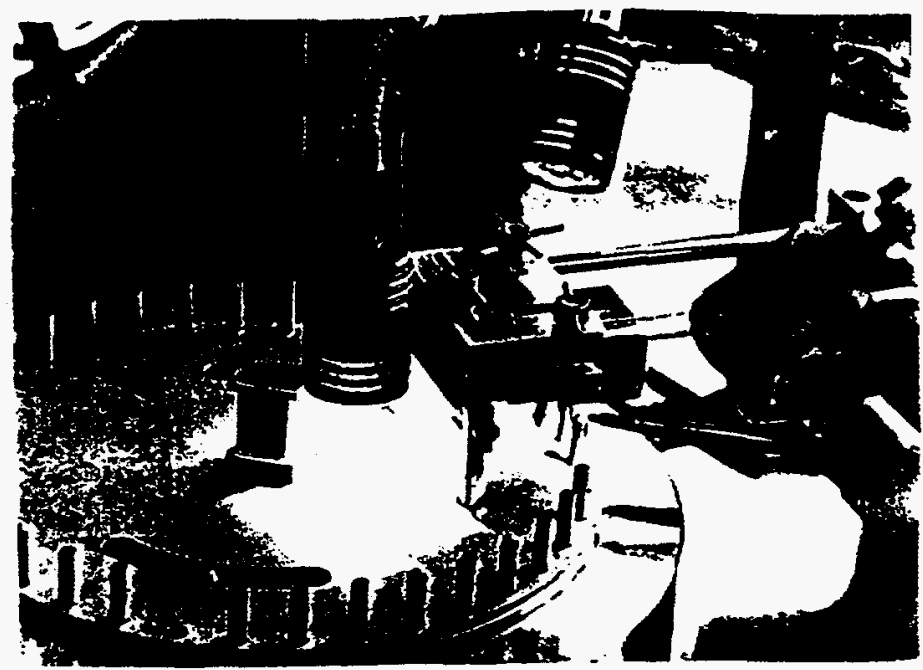

Figure 2. In-Cell Components of the Vision System.

images that facilitate the extraction of the edges of the dark fuel jacket from the light background and provides uniform gray levels both inside the jacket and in the background.

Figure 2 also shows the prism apparatus attached to the camera and located above the fuel jackets. The purpose of the prism apparatus is to provide a second view (top-view image) of the fuel jacket in addition to the frontview image directly produced by positioning the jacket in front of the camera. This is necessary since the jacket does not rotate axially and the camera is fixed in a given direction, thus producing only one front-view image. This configuration, however, limits the amount of information that can be extracted from welded elements and can preclude the detection of faulty welded elements when the anomaly occurs in the direction of the camera.

2. Out-Of-Cell Components. The in-cell tube-type camera of the vision system is connected to the out-of-cell components of the system located at the OCS. The main out-of-cell components of the vision system include a image processing computer, a host computer, and a video display monitor. The image processing computer is an Intelledex $386 \mathrm{HR}$ processor, 2 the host computer is a 386-based personal computer, and the video display monitor is a standard color monitor.

The image processing computer is connected to the in-cell camera, which when requested by the computer captures a video image of the fuel jacket and displays it on the video display monitor. The computer digitizes the image and through a library of functions detects the edges of the fuel jacket in both front- and top-view images, which are then analyzed for appropriate classification of the welded jacket. The host computer is mainly used for development of the software and storage of data files. 


\section{VISION SYSTEM MEASUREMENTS}

The automated visual inspection system classifies welded fuel jackets through a two-step process. First, with the vision system, it performs pre- and post-weld dimensional measurements (based on the detected edges) in both the front- and top-view images. Then, with the decision-making classifier, it analyzes the dimensional measurements and classifies the jackets as acceptable or unacceptable.

The bottom and top silhouettes in the left side of Fig. 3 show the front- and top-view images, respectively, captured by the in-cell camera and displayed by the video monitor during pre-weld inspection. The inserted end plug into the top of a jacket generates a front-view silhouette that resembles a triangle placed on top of a rectangle, and a top-view silhouette very close to a circle. The high contrast between the dark jacket and the light background and the homogeneous gray levels inside the two areas allow for the sharp detection of the edges of the jacket.

The right side of Fig. 3 shows the same two images captured during post-weld inspection. An acceptable weld, such as the one illustrated in the figure, generally shows a somewhat symmetric dome-like shape in the front-view image and a circular-like shape in the top-view image.

\section{A. Pre-Weld Inspection}

Pre-weld inspection is performed to achieve three main objectives: calibrate the post-weld top-view image, establish and fix the origin for the post-weld vertical measurements in the front-view image, and decrease the influence of the arrangement of the in-cell components of the vision system on the post-weld top-view measurements. The almost perfect circular image in the top-view of pre-weld jackets is used to calibrate, i.e., relate number of pixels to known real-world distances, for both top-view images. The uncertainty in the quality of welded jackets precludes the usage of landmarks in the post-weld image for performing the calibration. There is no need to fix the calibration of the front-view image since the undeformed edges of the jacket represented by the vertical lines of the silhouette are always present and can be used as calibration landmarks.

The lack of an image-dependent frame of reference for performing vertical measurements of the height of the welded dome (since each jacket can appear in a different vertical position) requires that we establish and fix the origin for vertical measurements based on the pre-weld image. For horizontal measurements, the left vertical edge of the silbouette is used as the horizontal origin. The pre-weld inspection is also used to decrease the influence of the arrangement of the in-cell components of
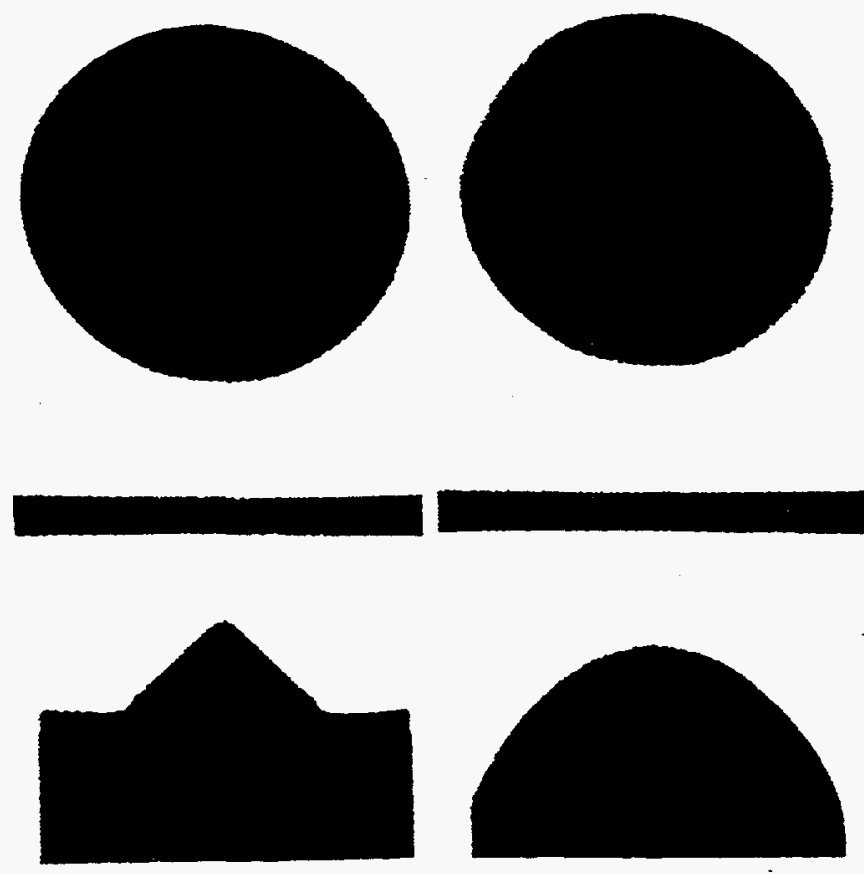

Figure 3. Front- and Top-View Images During Pre- and Post-Weld Inspections.

the vision system, such as the lighting scheme and alignment of the camera and prism apparatus, on the postweld top-view measurements. This is obtained by determining the $x$ and $y$ coordinates of 360 points along the edge of the circular pre-weld image and correlating these measurements with similar post-weld measurements to cancel out possible distortions.

\section{B. Post-Weld Inspection}

During the post-weld inspection, measurements are performed in both images which are later used to classify the welded jacket. In the front view, vertical measurements are performed at equidistant horizontal positions to determine the $y$ coordinate of 101 points along the edge of the dome of a welded jacket. In the top view, measurements are performed just like during the preweld inspection to determine the $\mathrm{x}$ and $\mathrm{y}$ coordinates of 360 points along the edge of the circular silhouette.

\section{STATISTICAL CLASSIFIER}

With the measurements performed during pre- and post-weld inspections, two decision-making classifiers are applied to classify welds as acceptable or unacceptable. Any classification technique requires, however, a set of user-specified criteria or feature variables that can be used to characterize the welds. In this section we describe the first classifier, a standard statistical process control classifier, and the criteria defined for classification. 


\section{A. Control Charts}

The statistical classifier (SC) is based on standard control chart techniques used in industrial quality control. ${ }^{3}$ Given samples containing measurements of feature variable $\mathrm{z}$ of known acceptable welds, a control chart is constructed by estimating the variable sample mean $\mathrm{E}(\mathrm{z})=$ $\overline{\mathbf{z}}$ and sample standard deviation $\sigma_{\mathrm{Z}}$ to obtain control intervals $\bar{z} \pm 3 \sigma_{z}$. This assumes that the parameter $z$ has a normal distribution with mean $\mu$ and variance $\sigma^{2}$, where $\mu$ and $\sigma$ can be estimated from the sample as $\bar{z}$ and $\sigma_{z}$, respectively. The $3 \sigma_{z}$ provides a confidence level of $99.74 \%$ that a measurement of feature variable $z$ will be located in the interval $\bar{z} \pm 3 \sigma_{z}$. Hence, if a future measurement of $z$ is found to be outside the limits $\bar{z} \pm$ $3 \sigma_{z}$ it is said to be "out of control" and is not accepted, otherwise, it is said to be "in control" and is accepted.

\section{B. Classification Criteria}

The classification criteria are designed based on the measurements performed during pre- and post-weld inspections described in Secs. III.A and III.B above, with the following two key underlying objectives. First, the criteria should be capable of detecting shape deformations characteristic of unacceptable welds such as welds with off-centered domes or "slumplers" caused by an eccentric electrode, welds with flat domes or "flat-tops" caused by too much energy deposition during welding, welds where the dome has expanded over the edge of the jacket or "mushrooms", as well as unwelded jackets or "no weld".

Second, the criteria should correlate the measurements performed during pre- and post-weld inspections, e.g., maximum post-weld jacket width given maximum pre-weld width, as well as measurements in the same or in the other image, e.g., fractional post-weld area of quadrants II and III in the top view given the height of the dome at the left intercept in the front view. Measurements of acceptable welds correlate well, which may not hold for unacceptable welds. The correlation in the measurements is represented in the criteria by defining conditional sample averages $E(z / w)$ and associated standard deviations, and using them in the control charts instead of the global sample mean $\bar{z}$ and $\sigma_{z}$. Here, the continuous conditional variable $w$ is discretized into intervals and the samples located inside each interval are used to estimate the conditional average and standard deviation.

Table I shows the 8 front-view criteria and 16 topview criteria used for the SC. In the top-view criteria, the radii of the top-view silhouette of both pre- and post-weld images are obtained by performing a least-squares fitting of the 360 points along the edge of the silhouette into a circle 4 and calculating the distances from the center of the fitted circle to the 360 points. The fitted circle is also divided into four $90^{\circ}$ quadrants starting at the right side of the circle and moving clock-wise on the top-view image.
Table I. Criteria for the Front- and Top-View Images.

\begin{tabular}{|c|}
\hline Front View Criteria \\
\hline 1. Dome left transition angle \\
\hline 2. Dome right transition angle \\
\hline $\begin{array}{l}\text { 3. Maximum dome height given the dome left } \\
\text { transition angle }\end{array}$ \\
\hline $\begin{array}{l}\text { 4. Maximum dome height given the dome right } \\
\text { transition angle }\end{array}$ \\
\hline $\begin{array}{l}\text { 5. Horizontal coordinate of the maximum dome } \\
\text { height given the dome left transition angle }\end{array}$ \\
\hline $\begin{array}{l}\text { 6. Horizontal coordinate of the maximum dome } \\
\text { height given the dome right transition angle }\end{array}$ \\
\hline $\begin{array}{l}\text { 7. Dome height at the left side of the jacket } \\
\text { given the dome left transition angle }\end{array}$ \\
\hline $\begin{array}{l}\text { 8. Dome height at the right side of the jacket } \\
\text { given the dome right transition angle }\end{array}$ \\
\hline $\begin{array}{c}\text { Top View Criteria } \\
\end{array}$ \\
\hline $\begin{array}{l}\text { 1. Maximum post-weld jacket width given the } \\
\text { maximum pre-weld width }\end{array}$ \\
\hline 2. Total post-weld area given total pre-weld area \\
\hline $\begin{array}{l}\text { 3. Fractional area of quadrants II and III given } \\
\text { the height of the dome at the left intercept }\end{array}$ \\
\hline $\begin{array}{l}\text { 4. Fractional area of quadrants I and IV given the } \\
\text { height of the dome at the right intercept }\end{array}$ \\
\hline $\begin{array}{l}\text { 5. Average post-weld radius of quadrant I given } \\
\text { its post-weld area }\end{array}$ \\
\hline $\begin{array}{l}\text { 6. Average post-weld radius of quadrant II given } \\
\text { its post-weld area }\end{array}$ \\
\hline $\begin{array}{l}\text { 7. Average post-weld radius of quadrant III given } \\
\text { its post-weld area }\end{array}$ \\
\hline $\begin{array}{l}\text { 8. Post-weld radius of quadrant IV given its } \\
\text { post-weld area }\end{array}$ \\
\hline $\begin{array}{l}\text { 9. Post-weld radial dispersion in quadrant I given } \\
\text { its average post-weld radius }\end{array}$ \\
\hline $\begin{array}{l}\text { 10. Post-weld radial dispersion in quadrant II } \\
\text { given its average post-weld radius }\end{array}$ \\
\hline $\begin{array}{l}\text { 11. Post-weld radial dispersion in quadrant III } \\
\text { given its average post-weld radius } \\
\end{array}$ \\
\hline $\begin{array}{l}\text { 12. Average post-weld radial dispersion in } \\
\text { quadrant IV given its average post-weld radius }\end{array}$ \\
\hline $\begin{array}{l}\text { 13. Post-weld radial error in quadrant I given its } \\
\text { pre-weld average radial enror }\end{array}$ \\
\hline $\begin{array}{l}\text { 14. Post-weld radial error in quadrant II given its } \\
\text { pre-weld average radial error }\end{array}$ \\
\hline $\begin{array}{l}\text { 15. Post-weld radial error in quadrant III given its } \\
\text { pre-weld average radial error }\end{array}$ \\
\hline $\begin{array}{l}\text { 16. Post-weld radial error in quadrant IV given its } \\
\text { pre-weld average radial enror }\end{array}$ \\
\hline
\end{tabular}




\section{NEURAL NETWORK CLASSIFIER}

A multilayer feedforward perceptron with a conjugate gradient-based backpropagation training algorithm is used as the artificial neural network classifier (NNC). 5,6 Computations with neural networks (NN) consist of two phases, training and recall. During the training phase, the network learns the mapping of $\mathrm{N}$-dimensional input vectors into $\mathrm{M}$-dimensional output vectors, after which, new input vectors are provided and the network is expected to recall the associated output vector. For classification purposes, the elements of the input vector are the feature variables used to characterize the weld, while the elements of the output vector represent the weld's class, e.g., acceptable or unacceptable.

The training of a NNC consists of partitioning the $\mathrm{N}$-dimensional feature space, spanned by the feature variables of the input vector, with decision boundaries or hyperplanes such that welds of a given class are separated from the other classes. ${ }^{7}$ Each processing unit or node in the first hidden layer of the NN can be associated with one decision boundary which, combined at the output layer, partitions the feature space into convex regions. A second hidden layer is necessary to partition the space into nonconvex and separated regions. For each classification problem there is a lower theoretical limit $L$ in the number of nodes in the first hidden layer required for successful separation of the training data, or equivalently, successful convergence of the training process. There is, however, a trade-off associated with the addition of nodes beyond $\mathrm{L}$. In one hand, the addition of nodes increases the number of decision boundaries which may decrease the misclassification rate of new welds during recall. On the other hand, as nodes are added, the network's ability to generalize beyond the training data decreases. If the number of nodes becomes too large, the network will "memorize" the training data by associating each training weld with one region of the space.

While having the same final objective, the statistical and neural network classifier achieve their goals by different means. The $S C$ is based only on a database of acceptable welds from which it calculates averages and standard deviations used to form the in-control intervals. In contrast, the database for the NNC must contain both acceptable and unacceptable welds in order for the network to find the appropriate decision boundaries that discriminate the two classes. This additional information allows NNCs to classify unacceptable welds at the failure mode level.

The NNC implicitly uses the interrelationships among all $\mathrm{N}$ feature variables of the input vector to classify welds. To account for relationships among variables in the $S C$, the user needs to specify which variables should be correlated and explicitly calculate the conditional averages $\mathrm{E}(\mathrm{z} \mid \mathrm{w})$. This can be time consuming, especially if one variable is thought to be correlated to two or more variables. On the other hand, the determination of the most appropriate $\mathrm{NN}$ architecture is also a time consuming task.

The NNC learns to classify welds by simultaneously partitioning an $\mathrm{N}$-dimensional feature space, populated with the training data, with nonlinear decision boundaries which, combined at the output node of the network, form decision regions or patterns for binary classification. The number of decision boundaries and shape of the partitioned feature space depends on the NN architecture and the type of mapping function of the nodes of the network. Through a similar interpretation (when the criteria are defined by global averages without accounting for correlations among the features), SCs classify welds by performing $\mathrm{N}$ independent one-dimensional partitionings in the feature variables. The number of decision boundaries is twice the number of criteria. They are located $3 \sigma$ away from the sample averages, and linearly partition the space into one hyperbox formed by the intersection of the $\mathbf{N}$ in-control intervals. Data points located inside the hyperbox are classified as acceptable, while data located outside are classified as unacceptable.

In spite of the differences in the two classification techniques, they have one key characteristic in common. Neither method will perform satisfactorily if there isn't enough information in the selected feature variables or criteria that are capable of distinguishing welds of distinct classes.

\section{CLASSIFICATION RESULTS}

To train, test, and compare the effectiveness of the two classifiers in accepting/rejecting acceptable and unacceptable welds, a total of 167 dummy stainless steel fuel element jackets were welded in a test facility outside the argon cell of the FCF. Of the 167 welds, 51 were labeled as unacceptable and the remaining 116 were labeled as acceptable based on visual inspections performed by an operator. The database of 51 unacceptable welds were purposely generated by depositing an incorrect amount of energy during the welding process, including the limiting case of no weld, and by forcing an eccentricity of the tungsten electrode towards the front, back, left, and right sides of the jacket. The 51 unacceptable welds consisted of 5 no welds, 25 front/back slumpers, 12 left/right slumpers, 3 flat-tops, and 6 of unspecified fault.

\section{A. Statistical Classifier}

Forty-four acceptable welds were used to obtain the statistics and associated in-control intervals for the 24 criteria in Table I. The remaining 72 acceptable (A) welds and all 51 unacceptable $(U)$ welds were used for testing the SC. The first row in Table II shows the results of the SC. Out of the 72 acceptable welds used for testing, 61 
Table II. Classification Results for the Statistical Classifier and Neural Network Classifier.

\begin{tabular}{|c|c|c|c|c|c|c|c|c|c|c|}
\hline \multirow{3}{*}{$\begin{array}{l}\text { Weld } \\
\text { Class }\end{array}$} & \multicolumn{2}{|c|}{ No. Welds Used for } & \multicolumn{8}{|c|}{ No. Welds Correctly Classified from the Testing Data } \\
\hline & \multirow[t]{2}{*}{ Training } & \multirow[t]{2}{*}{ Testing } & \multirow{2}{*}{$\begin{array}{l}\text { Statistical } \\
\text { Classifier } \\
\text { (SC) }\end{array}$} & \multicolumn{7}{|c|}{ Neural Network Classifier (NNC) } \\
\hline & & & & $19-11-1$ & $19-15-1$ & $19-17-1$ & $19-19-1$ & 19-23-1 & $5-5-1$ & $14-14-1$ \\
\hline $\begin{array}{l}\mathrm{A} \\
\mathrm{U}\end{array}$ & 44 & $\begin{array}{l}72 \\
51\end{array}$ & $\begin{array}{l}61 \\
45\end{array}$ & & & & & & & \\
\hline $\begin{array}{l}\mathrm{A} \\
\mathrm{U}\end{array}$ & $\begin{array}{l}44 \\
26\end{array}$ & $\begin{array}{l}72 \\
25\end{array}$ & & $\begin{array}{l}60 \\
16\end{array}$ & $\begin{array}{l}68 \\
14\end{array}$ & $\begin{array}{l}62 \\
16\end{array}$ & $\begin{array}{l}64 \\
16\end{array}$ & $\begin{array}{l}56 \\
16\end{array}$ & $\begin{array}{l}50 \\
14\end{array}$ & $\begin{array}{l}41 \\
15\end{array}$ \\
\hline $\begin{array}{l}A \\
U\end{array}$ & $\begin{array}{l}44 \\
13\end{array}$ & $\begin{array}{l}72 \\
38\end{array}$ & & & & & $\begin{array}{r}70 \\
8\end{array}$ & & & \\
\hline
\end{tabular}

were correctly classified as acceptable and 11 were incorrectly classified as unacceptable; and out of the 51 unacceptable welds, 45 were correctly classified and 6 were incorrectly classified as acceptable. Figure 4 illustrates the frequency in which each one of the 8 front-view criteria and 16 top-view criteria were used to correctly and incorrectly classify the 45 unacceptable (U/N) and the 11 acceptable (A/U) welds, respectively.

The misclassification of 11 acceptable welds is attributed to two major factors. The first one concerns the continuous adjustments of the in-cell components of the welding and vision systems, i.e., electrode, prism, and lighting scheme, which caused the top-view measurements used to define the top-view criteria to drift from configuration to configuration. This problem should be eliminated once the welding and vision systems are moved into the argon cell of the FCF and the final configuration of the components is kept fixed. The second factor concerns the much better resolution of the vision system as compared to the human eye. The lack of adequate resolution of the human eye could have labeled a weld as being acceptable, while some of its dimensions are quite distant from the average dimensions of the 44 acceptable welds used for statistics.

Five of the 6 unacceptable welds misclassified, were either front or back slumpers caused by an eccentricity of the electrode in the direction along the camera; a direction in which the camera is "blind" due to the lack of the depth perception. It seems that there isn't enough information in either the front- or top-view images to properly characterize front and back slumpers. Figure 5 illustrates this fact. The left picture shows the two images of a front slumper which look like a perfect weld, while the right picture shows the images of the same jacket after it was rotated $90^{\circ}$, in which case the fault is obvious. However, the occurrence of such a malfunction of the electrode is highly unlikely due to mechanical constraints in the welder. To simulate this unlikely failure mode, shims were deliberately placed in the welder to force an eccentricity of the electrode in the direction of the camera.

\section{B. Neural Network Classifier}

The NNC was trained and tested numerous times with varying $\mathrm{NN}$ architectures, input feature variables, and training data. In all cases, the number of layers of the network was fixed to three (one input, one hidden, and one output) with only one node in the output layer. The target values for acceptable and unacceptable welds were set to 0.90 and 0.10 , respectively, and a training convergence criterion of 0.01 was used. For the testing data, a threshold of 0.50 in the activation level of the output node was used for classification purposes. A weld

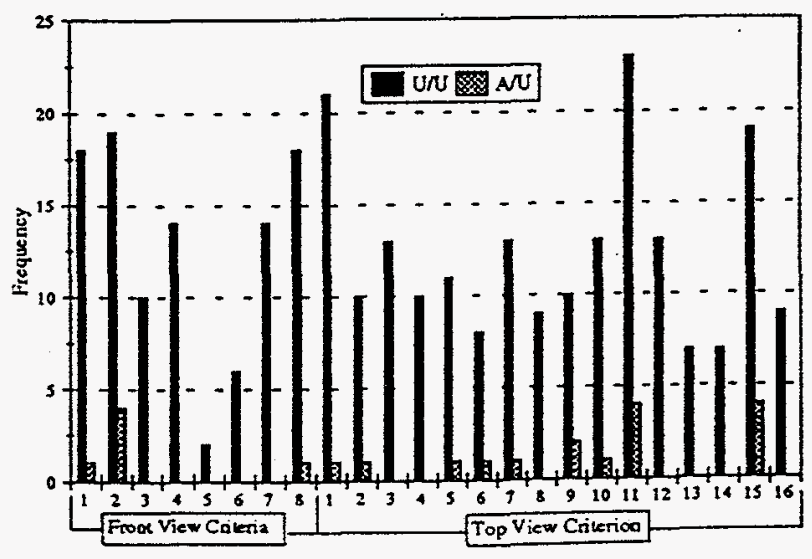

Figure 4. Frequency of the Criteria Used for Classification in the Statistical Classifier. 

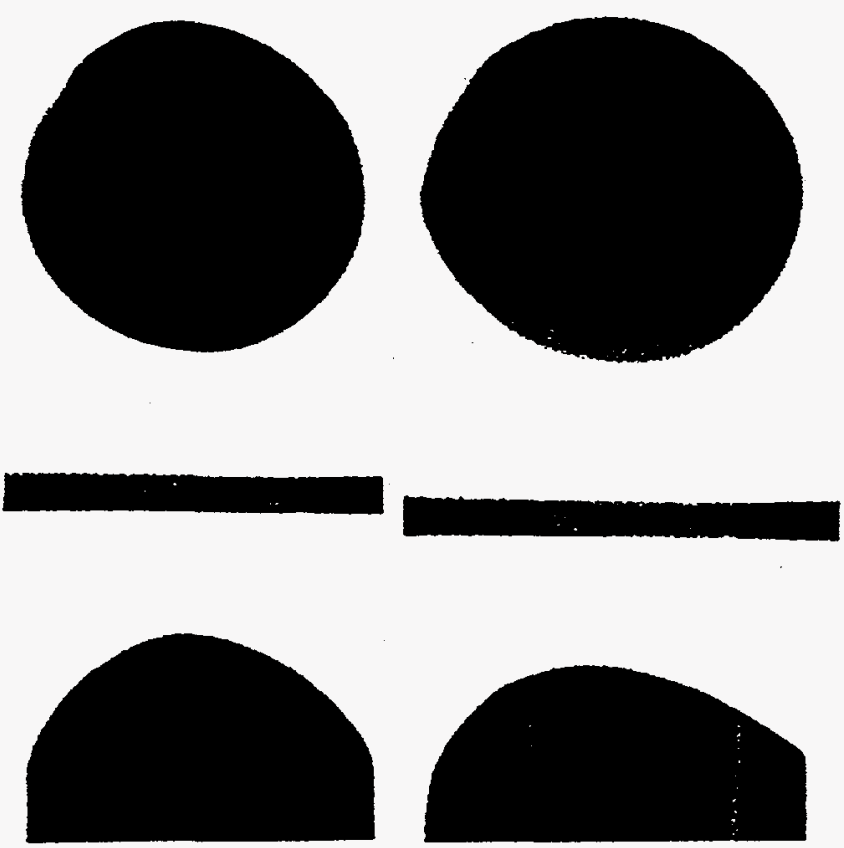

Figure 5. Front- and Top-View Images of a Front Slumper Before and After it is Rotated $90^{\circ}$.

with activation level above 0.5 was classified as acceptable, otherwise it was classified as unacceptable. The same 44 acceptable welds used for statistics in the SC were always used as the acceptable welds portion of the training data, and the 6 welds misclassified by the SC were also always used as part of the testing data. The feature variables input to the network were obtained from the criteria in Table I. However, instead of specifying which variables should be correlated through conditional averages, we provided the NNC with the feature variables and allowed the network itself to obtain the underlying correlations among the features during training.

1. Test 1. In the first test we fixed the number of input nodes or feature variables to 19 and tried 5 different number of nodes $(11,15,17,19$, and 23$)$ in the second layer or first hidden layer of the network. The 19 feature variables were obtained by discarding redundant and uninformative criteria in Table I, i.e., criteria that could be removed without affecting the performance of the SC. In the front view, 5 features $(1,2,4,7$, and 8$)$ were retained, while in the top view we retained $7(1,3,4,9,10,11$, and 12). To allow the NN to correlate pre- and post-weld measurements of the top view, we included the values of the 7 feature variables obtained during both pre- and postweld inspections for a total of $19(5+7+7)$ input variables. Of the 51 unacceptable welds in the database, 26 (3 no welds, 13 front/back slumpers, 6 leftright slumpers, 1 flat-top, and 3 of unspecified fault) were used for training and the remaining 25 for testing.
The second row in Table II shows the classification results obtained by changing the number of nodes in the second layer of the network. All five architectures, 19-11$1,19-15-1,19-17-1,19-19-1$, and 19-23-1, performed similarly in the classification of unacceptable welds, misclassifying either $9(25-19)$ or $11(25-14)$ welds. Like the 6 unacceptable welds misclassified by the SC, the majority of the unacceptable welds misclassified by the NNC were also either front or back slumpers. The slightly unfavorable comparison of the NN results is attributed to a lop-sided training database of 44 acceptable versus only 26 unacceptable welds, which bias the partition of the feature space towards the acceptable welds. In other words, it reserved a larger hypervolume to be associated with acceptable welds.

The influence of the number of nodes in the second layer in the performance of the NNC is more pronounced in the classification of acceptable welds. The number of correctly classified welds out of the 72 varied from 56 to 68 , and compared well with the 61 welds correctly classified by the SC. For the reasons presented in Sec.V, NN architectures with too few nodes in the first hidden layer, e.g., 19-11-1, or too many nodes, e.g., 19-23-1, did not perform as well as the other three networks, although they satisfactorily converged during the training phase.

2. Test 2. In the second test we separated the 5 features of the front-view image from the $14(7+7)$ features of the top-view image and trained two viewspecific networks with different architectures, 5-5-1 and 14-14-1, respectively. The classification results of these two networks are illustrated in the last two columns of Table II. In both cases these view-specific networks performed poorly. The poor performance of these networks is attributed to the lack of sufficient information in either front- or top-view images alone, and reinforces the importance of correlating measurements in both images. For instance, two welds, one acceptable and another unacceptable, could look alike in the front view but show large differences in the top view. When faced with welds of similar feature values belonging to different classes during training, the network will tend to "erroneously" partition the feature space in that region such that the training data can be separated. The lack of truly separable features causes this partition to be specific to the training data and to misclassify the test data.

3. Test 3. Analysis of the results of test 2 indicates that the cause for the misclassification of acceptable welds is the lack of sufficient information to correctly discriminate front/back slumpers from acceptable welds during training. In essence, during training we provide acceptable and front/back slumpers to the network with very similar feature values and ask it to classify them in opposite classes. 
To verify this hypothesis, in test 3 we removed all 13 front/back slumpers from the 26 unacceptable welds of the training data, and retrained the 19-19-1 network with 44 acceptable and 13 unacceptable welds. The 13 removed welds were added to the training data for a total of 38 unacceptable and 72 acceptable welds. The results of the NNC are illustrated in the third row of Table II. Now only 2 acceptable welds are misclassified out of 72 , while 30 unacceptable welds are misclassified including all 25 front/back slumpers of the database. The removal of the front/back slumpers from the training data prevented the network from "erroneously" partitioning the space in the region previously occupied by these slumpers allowing for correct classification of almost all acceptable welds in the test data.

The fact that all front/back slumpers were classified as acceptable welds show that indeed, based solely on the values of the 19 feature variables these welds seem to be acceptable welds. The feature variables do not contain enough information to discriminate this particular failure mode. The correct classification of this failure mode can only be done by providing information-rich features which when partitioned during training produce decision regions that can truly separate front/back slumpers from acceptable welds. The fact that 5 additional unacceptable welds with different failure modes were also misclassified is caused by the lop-sided training database with $\mathbf{4 4}$ acceptable and only 13 unacceptable welds that bias the partition of the feature space allocating a larger hypervolume to acceptable welds.

\section{CONCLUSIONS}

The overall results indicate that standard control chart techniques used in industrial quality control and multilayer feedforward neural networks provide comparable results in the classification of welded fuel jackets. The classification procedure of the two methods can be interpreted as the partition of a hyperspace spanned by $\mathrm{N}$ feature variables, in the case of the NNC, or N classification criteria, in the case of the SC, such that welds of a given class are separated from the other classes. The NNC partitions the $\mathrm{N}$-dimensional byperspace nonlinearly through a simultaneous search given a database of both acceptable and unacceptable welds, while the SC partitions the space linearly through $\mathrm{N}$ one-dimensional partitions based solely on acceptable welds.

Unlike the SC, the NNC intrinsically takes into account the possible relationships among all feature variables in the classification. In addition, by experimenting with different architectures and training data, the NNC allows us to gain insight of the classification problem and determine that the cause for misclassification of front/back slumpers is the lack of information-rich features capable of characterizing such failure mode. The performance of any classifier is highly dependent on the information content of the user-specified features. Ongoing work includes the design and testing of additional feature variables that are capable of characterizing this unlikely mode of failure.

\section{ACKNOWLEDGMENTS}

The authors wish to thank Ben Krause for collecting the data and Dale R. Wahlquist for discussions. This work was supported by the U.S. Department of Energy, Nuclear Energy Program, under contract W-31-109-ENG38.

\section{REFERENCES}

1. D. R. WAHLQUIST, "Automated Fuel Element Closure Welding System," Proc. ANS 5th Topical Meeting on Robotics and Remote Systems, Vol. II, P. 837, Knoxville, Tennessee, April 25-30, 1993.

2. Intelledex Vision Products, Wilsonville, Oregon, 97070 .

3. R. L. SCHEAFFER and J. T. McCLAVE, Statistics for Engineers, Duxbury Press, Boston, MA (1982).

4. B. B. CHAUDHURI and P. KUNDU, "Optimum Circular Fit to Weighted Data in Multi-Dimensional Space," Pattern Recognition Letters, 14, 1 (1993).

5. D. E. RUMELHART, G. E. HINTON, and R. J. WLLIAMS, "Learning Internal Representations by Error Propagation," Parallel Distributed Processing: Explorations in the Microstructure of Cognition, Vol. I, P. 319 , D. E. RUMELHART AND J. C. McCLELLAND, Eds., The MIT Press, Cambridge, Massachusetts (1986).

6. J. REIFMAN AND J. E. VITELA, "Accelerating Learning of Neural Networks with Conjugate Gradients for Nuclear Power Plant Applications," Nucl. Technol., 106, 225 (1994).

7. R. P. LIPPMAN, "An Introduction to Computing with Neural Networks," IEEE ASSP, 4, 2, 4 (1987). 